\title{
Respiratory complications in long-term survivors of oesophageal atresia
}

\author{
NICHOLAS E. DUDLEY and PETER D. PHELAN \\ From the Departments of Surgery and Thoracic Medicine, Royal Children's Hospital, \\ Melbourne, Australia
}

\begin{abstract}
Dudley, N. E., and Phelan, P. D. (1976). Archives of Disease in Childhood, 51, 279. Respiratory complications in long-term survivors of oesophageal atresia. One hundred of the 192 survivors of repaired oesophageal atresia at the Royal Children's Hospital, Melbourne, aged one year and over on 1 November 1973, were reviewed to determine the frequency of respiratory complications. 78 children suffered from more than 3 attacks of bronchitis per year during the first 3 years of life and $48 \%$ of the children aged over 8 years were having more than three attacks per year. Episodes of cough persisting longer than 2 weeks were also common. In the year preceding review, about half the children had more than 3 such episodes. 58 children had been admitted to hospital on at least one occasion for chest infection. It is suggested that recurrent inhalation of milk and food, consequent upon disordered oesophageal motility, is the major factor causing these respiratory complications.
\end{abstract}

The quality of life enjoyed by children after surgical correction of oesophageal atresia has received increasing attention in recent years now that the chances of survival are so favourable (Myers, 1974). Problems of dysphagia resulting from defective oesophageal motility and oesophageal stricture are well recognized, but surprisingly little has been written about the long-term respiratory complications. Laks, Wilkinson, and Schuster (1972), in a follow-up of 120 patients 15-25 years after operation at the Children's Hospital Medical Center, Boston, found that 14 of the 42 patients they were able to contact had respiratory problems: 12 complained of frequent colds and bronchitis and 2 had had recurrent episodes of pneumonia in recent years. Chrispin, Friedland, and Waterston (1966) reported 14 patients from The Hospital for Sick Children, London, who had frequent and distressing respiratory problems. Most were aged under 2 years and the respiratory difficulties were attributed to recurrent aspiration secondary to defective oesophageal motility.

This paper reports the frequency and severity of respiratory symptoms in a large group of children with repaired oesophageal atresia, followed for periods up to 20 years, and discusses possible predisposing factors. This is part of a larger study to assess the overall quality of life achieved by these children.

\section{Materials and methods}

One hundred patients from 192 survivors of repaired oesophageal atresia at this hospital aged one year and over on 1 November, 1973, were assessed. They included 62 males and 38 females and the ages at time of review are shown in Table I.

Details of the presentation, operative repair, and postoperative complications were obtained by review of the case notes. Each child, with mother (or guardian), was interviewed by one of us (N.E.D.) in order to obtain first-hand information of the child's subsequent course. Particular attention was given to symptoms

TABLE I

Age of patients at time of review

\begin{tabular}{c|c}
\hline Age (years) & No. \\
\hline $1-2$ & 11 \\
$2-3$ & 10 \\
$3-4$ & 4 \\
$4-5$ & 9 \\
$5-6$ & 5 \\
$6-7$ & 5 \\
$7-8$ & 3 \\
$8-9$ & 3 \\
$>9$ & 50 \\
\hline
\end{tabular}


associated with swallowing and those suggestive of recurrent lower respiratory inflammation. 42 patients had had studies of oesophageal motility using a pressurerecording device (Sensotec Model EMP3) consisting of three solid-state transducers incorporated in a flexible silicone probe. The results will be reported elsewhere.

\section{Results}

The incidence of recurrent bronchtis, defined as cough associated with some constitutional disturbance lasting for at least 5 days, is shown in Table II. 78 children suffered with more than 3 attacks of bronchitis per year during their first 3 years of life. 44 had more than 8 attacks per year in their first 3 years. The number of attacks of bronchitis decreased between the ages of 4 and 8 years, and after the age of 8 years only $48 \%$ of children were still having more than 3 attacks per year.

\section{TABLE II}

Recurrent bronchitis in all years up to review

\begin{tabular}{c|c|c|c}
\hline $\begin{array}{c}\text { No. of episodes } \\
\text { per year }\end{array}$ & $0-3$ years & $4-8$ years & $>8$ years \\
\hline $0-3$ & 22 & 18 & 52 \\
$4-8$ & 34 & 64 & 44 \\
$>8$ & 44 & 18 & 4 \\
\hline
\end{tabular}

Numbers expressed as percentages.

Mothers were asked more information about episodes of bronchitis in the year preceding review, in the belief that details of attacks in this period would be more accurately recalled. About half the episodes of bronchitis followed a coryzal illness, and about half were associated with fever. In approximately one-third of the children all episodes of bronchitis were associated with wheezing, and in another third wheezing occurred with some episodes. Vomiting was an uncommon symptom at the onset of illness and did not seem to be an initiating factor. Antibiotics were the usual therapy in about $90 \%$, but in over half the children there was a little improvement within 5 days of starting treatment.

Table III shows the incidence in the year before review of cough lasting more than 2 weeks and not associated with constitutional disturbances. In most children the cough was dry and brassy-the so-called 'TOF' cough. This was more of a problem in children under 8 years, but $5 \%$ of children over 8 years had more than 3 episodes in the preceding year. 15 children had between 1 and 3 episodes of pneumonia in the year before
TABLE III

Persistent cough in year before review

\begin{tabular}{c|c|c|c}
\hline No. of episodes & $0-3$ years & $4-8$ years & $>8$ years \\
\hline 0 & 24 & 8 & 19 \\
$1-3$ & 29 & 32 & 30 \\
$4-10$ & 18 & 35 & 40 \\
$>10$ or & 29 & 25 & 11 \\
virtually \\
continuous & & & \\
\hline
\end{tabular}

Numbers expressed as percentages.

review, and 1 child had five attacks. Half these children were aged less than 3 years, but 3 were older than 9 years. In all years up to review 58 children had been admitted to hospital at least once for an episode of chest infection, and 22 had been admitted on 3 or more occasions.

Recurrent bronchitis was not more common than expected in children who had lower respiratory complications in the early postoperative period. 44 patients had required oesophageal dilatation for stricture on at least one occasion, and all but one of these had recurrent bronchitis. 79 children had either dysphagia or difficulties with feeding, such as regurgitation of small amounts of food, trick movements during swallowing, or had to drink fluids during meals, and 65 of these (82\%) had suffered from recurrent bronchitis.

Results of barium swallow examination were available in 97 patients. In 42 it was reported as normal-but many examinations were limited to an assessment of oesophageal diameter at the site of anastomosis. The commonest abnormality was abnormal peristalsis associated with reflux of barium back into the oropharynx. This was present in one-third of patients. Pressure studies of oesophageal motility were abnormal in all 42 patients examined. Tracheal aspirate was examined for the presence of fat in 7 patients with recurrent bronchitis to try to find evidence of inhalation. 4 had numerous fat globules, and 3 occasional ones.

\section{Discussion}

This study confirmed the importance of symptoms of recurrent lower respiratory inflammation in children after repair of oesophageal atresia. Recurrent bronchitis, persistent cough, and pneumonia were all common, these disturbances being most common in the first 8 years of life, with a peak incidence for multiple episodes of bronchitis occurring before the age of 3 years. The frequency of bronchitis in these children is much greater than in normal children. Recurrent 
bronchitis associated with wheezing (asthma) occurs in about $20 \%$ of children (Williams and McNicol, 1969). A further $7 \%$ of children have 4 or more episodes of bronchitis per year unassociated with wheezing (Hall et al., 1972).

Suggestions have been made about the cause of bronchitis, but it seems most probable that it is due to recurrent inhalation of food, particularly milk. This appears to be consequent upon defective oesophageal motility, which predisposes to delayed emptying, regurgitation, and pooling of food in the oropharynx. Fat was present in the tracheal secretions aspirated from all 7 patients in whom the investigation was carried out, and this is highly suggestive of inhalation (Williams and Freeman, 1973). Defective oesophageal motility is extremely frequent after repair of oesophageal atresia, and is at least partly due to stripping of the vagal branches to the oesophagus. Dudley (1973) showed that piglets, after division of vagal branches to the intrathoracic oesophagus with or without a segmental resection of the oesophagus with reanastomosis, all developed recurrent inhalation with fat in tracheal aspirates, and had defective oesophageal motility. The fact that 43 of the 44 children with oesophageal stricture in this series also had recurrent bronchitis is likewise very suggestive that inhalation is a factor. While there was not a statistically significant association between feeding difficulties and recurrent bronchitis, both were extremely common in this group of children. Children who had postoperative respiratory complications were not found to have an increased number of episodes of recurrent bronchitis, unlike the experience reported by Laks et al. (1972). However, in both series an association between oesophageal stricture and recurrent bronchitis was found.

Viral respiratory infections may have been important in precipitating episodes of bronchitis because of the frequent association with coryzal symptoms. The incidence of bronchitis, however, is much greater than could have been expected from viral infection alone. Wheezing was also frequent in younger children, but this may simply indicate widespread bronchial and bronchiolar obstruction (Phelan, 1972) and is not necessarily evidence of asthma. 13 of the 100 patients had first-degree relatives with asthma, which is about the expected percentage in the community (Williams and $\mathrm{McNicol}, 1969)$, and 10 of these had frequent bronchitis.

Tracheomalacia has been suggested as an important factor in causing recurrent cough after repair of oesophageal atresia (Glasson, 1974).
Though this is unlikely to produce coughing without bronchitis, it could be a factor in the development of wheezing. Tracheomalacia will result in expiratory collapse of the trachea only in the presence of positive pleural pressure during expiration. Positive pleural pressure is present during quiet expiration only if there is widespread disease of the more peripheral airways such as that caused by inhalation. It is dangerous to make a diagnosis of tracheomalacia solely on narrowing of tracheal diameter during expiration because quite marked narrowing of the normal trachea can occur in the presence of widespread peripheral obstruction (Wittenborg, Gyepes, and Crocker, 1967). It is very likely that bronchitis caused by inhalation is responsible for much of the tracheal narrowing attributed to tracheomalacia.

Emery and Haddadin (1971) found squamous metaplasia in the trachea or bronchi of $80 \%$ of infants dying in the early postoperative period. They suggested that the presence of nonciliated squamous epithelium in the major respiratory passages would seriously impair the anti-infection mechanism of the lung and hence could contribute to repeated attacks of bronchitis. While these authors suggested that the epithelial changes were probably an associated congenital anomaly, they could also be the result of repeated inhalation of gastric contents.

This study has focused attention on the possible sequelae of impaired oesophageal motility, but it is not conclusive that inhalation is the major factor in causing recurrent respiratory problems. The study was retrospective and sampling of tracheal aspirates was not possible on many patients. A careful prospective study with detailed investigation of patients with recurrent bronchitis is needed. Investigations should include study of oesophageal and tracheal function, tracheal aspirate, and immunological factors.

If inhalation is the main cause of these respiratory problems, prevention may be possible if vagal branches to the oesophagus can be preserved at the time of repair. The Duhamel reconstruction of the oesophagus (Sulamaa, Gripenberg, and Ahvenainen, 1951-1952; Berman and Berman, 1953; and Duhamel, 1957) popularized by Beardmore (Ty, Brunet, and Beardmore, 1967), has shown the feasibility of this approach, which involves minimal mobilization. More work is necessary, and there may be other facets in the disordered oesophageal motility. Inhalation can be minimized by feeding infants thickened feeds, keeping them in a semiupright position for 1-2 hours after feeds, and by giving them a small drink 
of water after feeds. Children should also be encouraged to have a drink of water after meals and should not go to bed within 1-2 hours of eating or drinking.

The advice and encouragement of Mr. N. A. Myers, Mr. M. Kent, and Miss Helen Noblett are gratefully acknowledged. The patients studied were under their care.

\section{REARERNCES}

Berman, J. K., and Berman, E. J. (1953). Congenital atresia of the esophagus with tracheo-esophageal fistula. A simplified technique of restoring continuity of the esophagus. American Fournal of Surgery, 86, 436.

Chrispin, A. R., Friedland, O. W., and Waterston, D. J. (1966). Aspiration pneumonia and dysphagia after technically successful repair of oesophageal atresia. Thorax, 21, 104.

Dudley, N. E. (1973). Inhalation (aspiration) pneumonia following experimentally induced disordered motility of the oesophagus. Australian Paediatric fournal, 8, 289.

Duhamel, B. (1957). Technique Chirurgicale Infantile. Masson, Paris.

Emery, J. L., and Haddadin, A. J. (1971). Squamous epithelium in the respiratory tract of children with tracheo-oesophageal fistula. Archives of Disease in Childhood, 46, 236.

Glasson, M. (1974). Tracheomalacia in association with tracheooesophageal fistula. Australian Paediatric fournal, 10, 238.
Hall, G. J. L., Gandevia, B., Silverstone, H., Searle, J. H. A., and Gibson, H. B. (1972). The interrelationship of upper and lower respiratory tract symptoms and signs in seven year old children. International fournal of Epidemiology, 1, 389.

Laks, H., Wilkinson, R. H., and Schuster, S. R. (1972). Longterm results following correction of esophageal atresia with tracheo-esophageal fistula. Fournal of Pediatric Surgery, 7, 591.

Myers, N. A. (1974). Oesophageal atresia. The epitome of modern surgery. Annals of the Royal College of Surgeons of England, 54, 277.

Phelan, P. D. (1972). Wheezing in Childhood. Australian Paediatric Fournal, 8, 167.

Sulamaa, M., Gripenberg, L., and Ahvenainen, E. K. (1951-1952). Prognosis and treatment of congenital atresia of the oesophagus. Acta Chirurgica Scandinavica, 102, 141.

Ty, T. C., Brunet, C., and Beardmore, H. E. (1967). A variation in the operative technique for the treatment of esophagea atresia with tracheoesophageal fistula. Fournal of Pediatric Surgery, 2, 118.

Williams, H. E., and Freeman, M. (1973). Milk inhalation pneumonia-the significance of fat filled macrophages in tracheal secretions. Australian Paediatric fournal, 9, 286.

Williams, H. E., and McNicol, K. N. (1969). Prevalence, natural history and relationship of wheezy bronchitis and asthma in children. An epidemiological study. British Medical fournal, 4, 321.

Wittenborg, M. H., Gyepes, M. T., and Crocker, D. (1967). Tracheal dynamics in infants with respiratory distress, strido and collapsing trachea. Radiology, 88, 653.

Correspondence to Mr. N. E. Dudley, Radcliffe Infirmary, Oxford OX2 6HE. 